\title{
Correlation between Pokemon and MDM2 in Carcinogenesis of Lung Squamous Cell Carcinoma in Rats \\ WY Fan ${ }^{1,3}, \mathrm{JN} \mathrm{Jiao}^{1}, \mathrm{~K} \mathrm{Guo}^{2}, \mathrm{ZP} \mathrm{Xu}{ }^{3,4}, \mathrm{JT} \mathrm{Lin}^{3,4}, \mathrm{XW} \mathrm{Wang}^{3,4}, \mathrm{JY} \mathrm{Fan}^{3,4}, \mathrm{CQ}$ Yang $^{3,4}$
}

\begin{abstract}
This study was designed to investigate correlation between proto-oncogene POK erythroid myetoid ontogenic factor (Pokemon) and oncogene MDM2 in carcinogenesis of lung squamous cell carcinoma in rats. Protein and mRNA expressions of Pokemon and MDM2 in different stages of rat lung squamous cell carcinoma were measured by immunochemistry staining and in situ hybridization assays. Lung squamous cell carcinoma could be viewed under microscope in 60 rats (success rate: $80 \%$ ) after treatment with carcinogen. Among these rats, 21 ones were with bronchial epithelial hyperplasia, 13 ones were with atypical hyperplasia, 28 ones were with carcinoma in situ, 20 ones had invasive carcinoma and 16 ones had metastatic carcinoma. There were significant differences of Pokemon and MDM2 expression between control group and atypical hyperplasia group or squamous cell carcinoma group $(P<0.05)$. There were also significant differences of both genes between non-metastatic carcinoma group and metastatic carcinoma group $(P<0.05)$. Pokemon expression was positively correlated with MDM2 expression $(\mathrm{r}=0.616, P=0.000)$. These findings indicate that Pokemon and MDM2 were highly expressed in rat lungs following carcinogenesis of lung squamous cell carcinoma. The expression of Pokemon and MDM2 may contribute to genesis and development of lung squamous cell carcinoma in rats.
\end{abstract}

Keywords: In situ hybridization, lung cancer, lung squamous cell carcinoma, MDM2, Pokémon

From: ${ }^{1}$ College of Basic Medicine, Xinxiang Medical University, Xinxiang 453003, Henan, China ${ }^{2}$ The Third Affiliated Hospital of Xinxiang Medical University, Xinxiang 453003, Henan, China. ${ }^{3}$ College of Life Sciences, Xinxiang Medical University, Xinxiang 453003, Henan, China. ${ }^{4}$ Key Laboratory of Henan Province for Medical Tissue Regeneration, Xinxiang Medical University, Xinxiang 453003, Henan, China

Correspondence: Dr Z Xu, College of Life Sciences, Xinxiang Medical University, Xinxiang 453003 Henan, China. E-mail: zhenpingxuxx@126.com

*These authors contributed equally to this work. 


\section{INTRODUCTION}

The development and metastasis of tumors are a complex process that involves in expression of serial genes. The activation of pro-oncogenes and inactivation of tumor suppressor genes are important contributory mechanisms underlying tumor progression and metastasis (1). Detection of tumor markers is a rapid, sensitive and non-invasive technique that offers a novel approach for early diagnosis of the genesis of lung cancers (2). Many genes and microRNAs have been identified as biomarkers of lung cancers (3-5). Their expression levels in the lung tissue, and even in blood can indicate the genesis and development of certain kinds of lung cancers.

Pokemon (POK erythroid myetoid ontogenic factor) is one of the members of POK protein family, which is encoded by Zbtb7 gene (6). A recent study demonstrated that Pokemon was a pro-oncogene that enables cancer cells to resist aging and death, and participated in regulating the expression and activity of other pro-oncogenes and oncogenes (6). Pokemon was upregulated following lymphomagenesis (6). The following studies demonstrated that Pokemon was expressed in lung cancers tissues and relevant to genesis of lung cancers $(7,8)$. MDM2 (Mouse double minute 2 homolog) is a protein that is encoded by the MDM2 gene. MDM2 is a negative regulator of the p53 tumor suppressor. MDM2 expression and activation inhibit P53 transcriptional activity and the following functions, and in turn P53 also negatively regulates MDM2 expression and activity, which forms a negative feedback loop $(9,10)$. The expression of MDM2 has also been related to the development of lung cancers (11).

Although Pokemon and MDM2 both have been viewed as biomarkers of lung cancers, their expressions and correlations in different stages of lung squamous cell carcinoma have not been elucidated. In this study, we investigated Pokemon and MDM2 expressions and correlations following the development of lung squamous cell carcinoma in 
rats.

\section{MATERIALS AND METHODS}

Animals

Ninety Wistar rats (male/female: 1/1) were purchased from Experimental Animal Center of Henan Province. Methylcholanthrene $(442,388)$ and diethyl nitrosamine $(73,861)$ were obtained from Shanghai Sigma Company (Shanghai, China). Lung squamous cell carcinoma rat models were established as previously described (12). Rats were randomly divided into two groups: tumor group (75 animals) and control group (15 animals). The rats in tumor group were treated with carcinogen, and the rats in control group were treated with iodized oil. At day 30, 60, 90, 120, and 180 following treatment with drug, the rats (15 animals in tumor group and 3 animals in control group/each time) were sacrificed, and tissues from the perfusion sites were collected. Based on the pathological grades, rats in tumor group were divided into: atypical hyperplasia, squamous cell carcinoma, non-metastatic carcinoma and metastatic carcinoma groups.

\section{In situ hybridization}

The Pokemon and MDM2 in situ hybridization kit, Pokemon and MDM2 hybridization in situ kit as well as rabbit anti mouse polyclonal antibody kit, BCIP/NBT color kit and DAB chromogenic kit were purchased from Wuhan Boster company. Tissues were dehydrated, embedded and made into slices according to standard protocols. After inactivation of endogenous enzyme, the slices were incubated with freshly diluted $3 \%$ citric acid pepsin for $20 \mathrm{~min}$. And subsequently, the slices were incubated with pre-hybridization solution at $38^{\circ} \mathrm{C}$ for $4 \mathrm{~h}$, followed by incubation with hybridization solution at $40^{\circ} \mathrm{C}$ for $15 \mathrm{~h}$. After washing with PBS, the slices were incubated with mouse anti-digoxin for $120 \mathrm{~min}$, and then treated 
with streptomycin affinity peroxidase complex $(\mathrm{SABC})$ and biotin peroxidase for $5 \mathrm{~min}$ at room temperature. After washing, the slices were re-stained with hematoxylin, and then mounted.

\section{Immunochemistry staining}

Pokemon and MDM2 immunochemistry kits, as well as rabbit anti-mouse polyclonal antibody kit were purchased from Wuhan Boster company. Slices were dewaxed, heated in a microwave for 20 min to repair antigens, and then treated with $3 \% \mathrm{H}_{2} \mathrm{O}_{2}$ for 10 min to eliminate endogenous peroxidase activity. After blocking with serum, the slices were incubated with rabbit anti mouse polyclonal antibody $(1: 100)$ at $4{ }^{\circ} \mathrm{C}$ overnight. After washing 3 times with PBS, the slices were incubated with secondary antibody, and then horseradish peroxidase. Subsequently, the slices were stained by DAB solution, and re-stained by hematoxylin.

\section{Semiquantitative score analysis}

Pokemon and MDM2 mRNA expression was analyzed by calculating the blue/purple particles in the cytoplasm as previously reported (13). More than 100 cells and at least five high-resolution fields in each slice were analyzed. The average percentage of positive staining was calculated based on the numbers of positive cells vs. total cells in the slices. Grades are as follows: $0-5 \%$ - 0 point, $6-25 \%-1$ point, $26-50 \%-2$ points, $51-75 \%-3$ points, and $>75 \%$ - 4 points. Staining degrees were quantified based on staining colors and intensity: uncolored staining was viewed as 0 point, purple/blue positive staining was viewed as 1 point, brown/blue positive staining was viewed as 2 points, and deep blue/purple staining was viewed as 3 points. The final score results were positive staining grades plus staining degrees: 0-1 was negative $(-), 2-3$ was weakly positive $(+), 4-5$ was moderately positive $(++)$, and 6-7 was strong positive $(+++)$. 


\section{Statistical analysis}

Data were presented as mean \pm standard deviation, and analyzed by $\chi^{2}$ test. Statistical difference between two groups was analyzed by $q$ test. Correlation analysis between Pokemon and MDM2 was performed by using Pearson correlation analysis. All data were statistically analyzed by SPSS 17.0 software.

\section{RESULTS}

\section{Pathological changes of lung squamous cell carcinoma in rats}

Normal bronchi, invasive carcinoma, and low differentiated squamous carcinoma can been seen in rat lungs following HE staining (Fig. 1A and B). In some slices, different stages of carcinogenesis such as bronchial epithelial hyperplasia, squamous metaplasia, dysplasia, carcinoma in situ, invasion and poorly differentiated carcinoma coexisted in the same fields (Fig. 1C). Lung squamous cancer, lymph node metastasis and pleural invasion simultaneously appeared in rat lungs at day 120 and 180 (data not shown). Sixty (of 75) rats were suffered from lung squamous cell carcinoma, including 21 cases with bronchial epithelial hyperplasia, 13 cases with dysplasia, 28 cases with carcinoma in situ, 20 cases with invasive cervical cancer and 16 cases with metastatic carcinoma. Of note, multiple phases coexisted in the same rat cancer lesions.

\section{Pokemon Expressions in Different Stages of Carcinoma Tissues}

Pokemon mRNA was mainly localized in cytoplasm with scattered or diffused distribution (Fig. 2A and B), but proteins were mainly located in the nucleus (Fig. 2C and D). Semi-quantitative score analysis indicated that score of Pokemon mRNA $n$ in normal bronchial epithelium (control group) was $1.25 \pm 1.03$, while the scores in hyperplasia, atypical 
hyperplasia and squamous cell carcinoma groups were $2.11 \pm 0.79,2.88 \pm 1.10$ and $4.17 \pm 1.05$, respectively. The variance among all groups was $F=31.454(P<0.05)$. The differences between control group and atypical hyperplasia group or squamous cell carcinoma group were statistically significant $(P<0.05)$, while there was no significant difference between control group and hyperplasia group, as well as between hyperplasia group and atypical hyperplasia group $(P>0.05)$. Overall, Pokemon expression in control group was lower than in all carcinogenesis groups. The score of Pokemon mRNA expression in metastatic carcinoma group was also significantly higher than in non-metastatic carcinoma group $(P<$ 0.05, Table 1).

\section{MDM2 expressions in different stages of carcinoma tissues}

MDM2 mRNA and protein both were localized in nucleus with scattered or diffused distribution (Fig. 3A-D). The score of MDM2 expression in normal bronchial epithelium (control group) was $1.21 \pm 0.74$, while the scores in hyperplasia, atypical hyperplasia and squamous carcinoma groups were $2.09 \pm 0.76,3.03 \pm 1.00$ and $4.17 \pm 1.18$, respectively. The variance among all groups was $F=30.669(P<0.05)$. The difference between control group and squamous cell carcinoma group was statistically significant $(P<0.05)$, while the differences between normal control group and hyperplasia group, as well as between hyperplasia group and atypical hyperplasia group was not significant $(P>0.05)$. MDM2 expression in control group was also lower than in all carcinogenesis groups. The difference between the metastatic cancer and non-metastatic cancer groups was statistically significant $(P<0.05$, Table 1)

Correlation between $m R N A$ expressions of Pokemon and MDM2 in rat lung squamous cell carcinoma. The ratio of MDM2 mRNA positive/Pokemon mRNA positive in lung tissues was $86.84 \%$, while the ratio of MDM2 mRNA negative/Pokemon mRNA negative was $24.24 \%$, indicating a positive correlation between these two genes $(r=0.616, \chi 2=41.362$, 
$P=0.000$, Table 2).

\section{DISCUSSION}

Pokemon gene is located in the third subzone of the first zone in the short arm of human chromosome 19 (19P13.3). The gene contains two exons and two introns, and encodes a 155 amino acid protein with a highly conserved protein-protein interaction domain (BTB domain) in the N-terminal and zinc finger structure in the C-terminal (14). Guo et al. (15) found that Pokemon mRNA level was much higher in bladder cancer tissues than in normal tissues. Other studies also indicated a high expression of Pokemon in lung cancers $(7,8)$. These studies suggest a possibility to diagnose cancers by detecting Pokemon gene expression (16).

To investigate expression pattern of Pokemon gene following the development of lung cancer, we established a lung squamous cell carcinoma rat model, and analyzed Pokemon expression using in situ hybridization in lung squamous cell carcinoma and adjacent tissues. Our results revealed a significant difference in Pokemon expression between control group and atypical hyperplasia- or squamous cell carcinoma-group $(P<0.05)$, accompanied by a gradually increased expression trend following the progression of lung cancer. The pathological score of Pokemon expression in metastatic carcinoma tissues was higher than in non-metastatic tissues. These results were consistent with previous reports by Zhao et al., which showed a high expression of Pokemon at gene and transcription levels $\mathrm{n}$ in human non-small cell lung cancer, but low or no expression in normal and adjacent tissues (7).

MDM2 is an oncogene identified in BALB/c mice cell lines, and located in the 12q13-14 chromosome region. Under the physiological conditions, MDM2 is induced by wild-type P53 and promotes the degradation of P53 (17). Our results showed that MDM2 
expression was higher in atypical hyperplasia and squamous cell carcinoma groups than in control group. Of note, MDM2 expression was gradually increased following the progression of lung cancer in rats. Like Pokemon, pathological score of MDM2 was also higher in metastatic carcinoma tissues than in non-metastatic tissues. These results indicate that expression of MDM2 gene involves in the development and progression of squamous cell carcinoma in rats.

We also analyzed the correlation between Pokemon and MDM2 mRNA expression in squamous cell carcinomas by in situ hybridization. Our results demonstrated a positive correlation between expressions of these two genes $(\mathrm{r}=0.616, P=0.000)$. This suggests that MDM2 and Pokemon expression are mutually reinforced in the development of lung squamous cell carcinoma. Our findings indicate that Pokemon and MDM2 both involves in the development and metastasis of lung squamous cell carcinoma, and simultaneous intervention of both genes may be a better strategy to prevent and treat lung cancers.

\section{ACKNOWLEDGEMENTS}

This study was supported by the Key Project of Education Department, Henan Province Science and Technology (13A320866). 


\section{REFERENCES}

1. Zhang J, Chen $\mathrm{YH}$ and $\mathrm{Lu}$ Q. Pro-oncogenic and anti-oncogenic pathways: opportunities and challenges of cancer therapy. Future Oncol 2010; 6: 587-603.

2. Zeng GQ, Zhang PF, Deng X, Yu FL, Li C, Xu Y et al. Identification of candidate biomarkers for early detection of human lung squamous cell cancer by quantitative proteomics. Mol Cell Proteomics 2012; 11: M111.

3. Tufman A, Huber RM. Biomarkers in lung cancer: A clinician's perspective. Cancer Biomark 2010; 6: 123-35.

4. Hennessey PT, Sanford T, Choudhary A, Mydlarz WW, Brown D, Adai AT et al. Serum microRNA biomarkers for detection of non-small cell lung cancer. PLoS One 2012; 7: e32307.

5. Boeri M, Pastorino U, Sozzi G. Role of microRNA in lung cancer: microRNA signatures in cancer prognosis. Cancer J 2012; 18: 268-74.

6. Meada T, Hobbs RM, Merghoub H, Guernah I, Zelent A, Cordon-Cardo C et al. Rde of the proto-onco-gene Pokémon in cellular transformation and ARF repression. Nature 2005; 433: 278-85.

7. Zhao ZH, Wang SF, Yu L, Wang J, Chang H, Yan WL et al. Expression of transcription factor Pokemon in non-small cell lung cancer and its clinical significance. Chin Med J (Engl) 2008; 121: 445-9.

8. Cui J, Meng X, Gao X, Tan G. Curcumin decreases the expression of Pokemon by suppressing the binding activity of the Sp1 protein in human lung cancer cells. Mol Biol Rep 2010; 37: 1627-32. 
9. Qiao D, Gaitonde SV, Qi W, Martinez. Deoxychidic acid suppresser p53 by stimul-ating proteasome-mediated p53 protein degradation. Carcinogenesis 2001; 22: 957-64.

10. Pamia G, Filiberti L, Vikhanshaya F, Carrassa L, Taya Y, D'incalci M et al. Cisplatinum and taxol induce different patterns of p53 phosphorulation. Neoplasia 2001; 3: 10-6.

11. Zhang DH, Zhang LY, Liu DJ, Yang F, Zhao JZ. Expression and significance of MMP-9 and MDM2 in the oncogenesis of lung cancer in rats. Asian Pac J Trop Med 2014; 7: 585-8.

12. Faridi A1, Rudlowski C, Biesterfeld S, Schuh S, Rath W, Schröder W. Long-term follow-up and prognostic significance of angiogenic basic fibroblast growth factor (bFGF) expression in patients with breast cancer. Pathol Res Pract 2002; 198: 1-5.

13. Jiang X, Zhou JH, Deng ZH, Qu XH, Jiang HY, Liu Y. Expression and significance of Notch1, Jagged1 and VEGF in human non-small cell lung cancer. Zhong Nan Da Xue Xue Bao Yi Xue Ban 2007; 32: 1031-6.

14. Collins T, Stone JR, Williams AJ. All in the family: the BTB/PO2, KR-AB, and SCAN Domains. Mol Cell Biol 2001; 21: 3609-15.

15. Guo C, Zhu K, Sun W, Yang B, Gu W, Luo J et al. The effect of Pokemon on bladder cancer epithelial-mesenchymal transition. Biochem Biophys Res Commun 2014; 443: $1226-31$.

16. He S, Liu F, Xie Z, Zu X, Xu W, Jiang Y. P-Glycoprotein/MDR1 regulates pokemon gene transcription through p53 expression in human breast cancer cells. Int J Mol Sci 
2010; 11: 3309-51.

17. Orre LM, Panizza E, Kaminskyy VO, Vernet E, Gräslund T, Zhivotovsky B et al. S100A4 interacts with p53 in the nucleus and promotes p53 degradation. Oncogene 2013; 32: 5531-40. 
Table 1: Scores of Pokemon and MDM2 mRNA expression in normal and different stages of tumor tissues $(\bar{x} \pm s)$

\begin{tabular}{lll}
\hline Groups & Pokemon mRNA & MDM2 mRNA \\
\hline Normal control & $1.25 \pm 1.03$ & $1.01 \pm 0.74$ \\
hyperplasia & $2.11 \pm 0.79$ & $2.09 \pm 0.76$ \\
Atypical hyperplasia & $2.68 \pm 1.10^{*}$ & $3.03 \pm 1.00^{*}$ \\
squamous carcinoma & $4.17 \pm 1.05^{*}$ & $4.17 \pm 1.18^{*}$ \\
Non-metastatic carcinoma & $3.64 \pm 1.11^{\#}$ & $3.79 \pm 1.15^{\#}$ \\
metastatic carcinoma & $4.53 \pm 0.86$ & $4.59 \pm 0.93$ \\
\hline
\end{tabular}

${ }^{*} \mathrm{p}<0.05,{ }^{\triangle}{ }_{\mathrm{p}}<0.05,{ }^{\triangle} \mathrm{p}<0.05$ vs. normal control group; ${ }^{*} \mathrm{p}<0.05$ vs. metastatic carcinoma group.

Table 2: Correlation of Pokemon and MDM2 mRNA expressions in lung tissues

\begin{tabular}{|c|c|c|c|}
\hline \multicolumn{2}{|c|}{ Pokémon mRNA } & \multicolumn{2}{|c|}{ MDM2 mRNA } \\
\hline & $n$ & + & - \\
\hline+ & 76 & 66 & 10 \\
\hline- & 33 & 8 & 25 \\
\hline Total & 109 & 74 & 35 \\
\hline
\end{tabular}



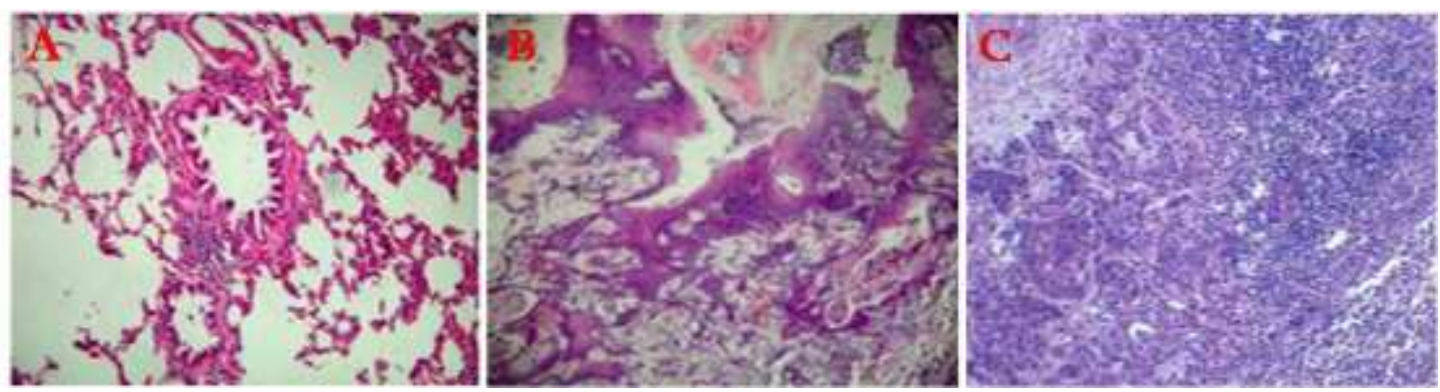

Fig 1: HE staining shows different stages of lung carcinoma in rats. A. Normal bronchus $(\times 200)$; B. invasive carcinoma $(\times 200)$; C. Low differentiated squamous carcinoma.
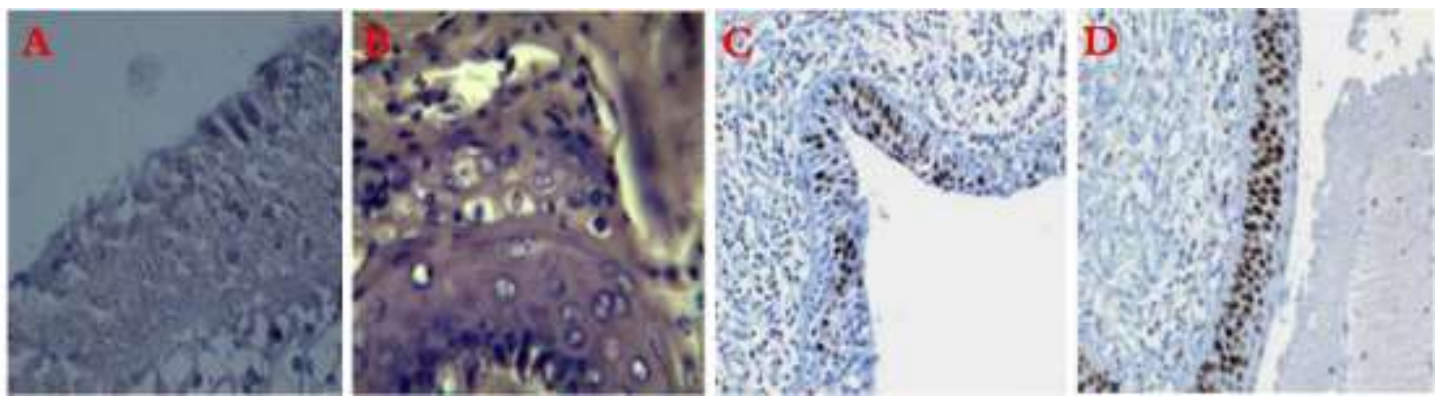

Fig. 2: In situ hybridization assays show Pokemon mRNA and protein expressions in rat lung tissues. A and B. Pokemon mRNA expression $(\times 400)$; C and D. Pokemon protein expression
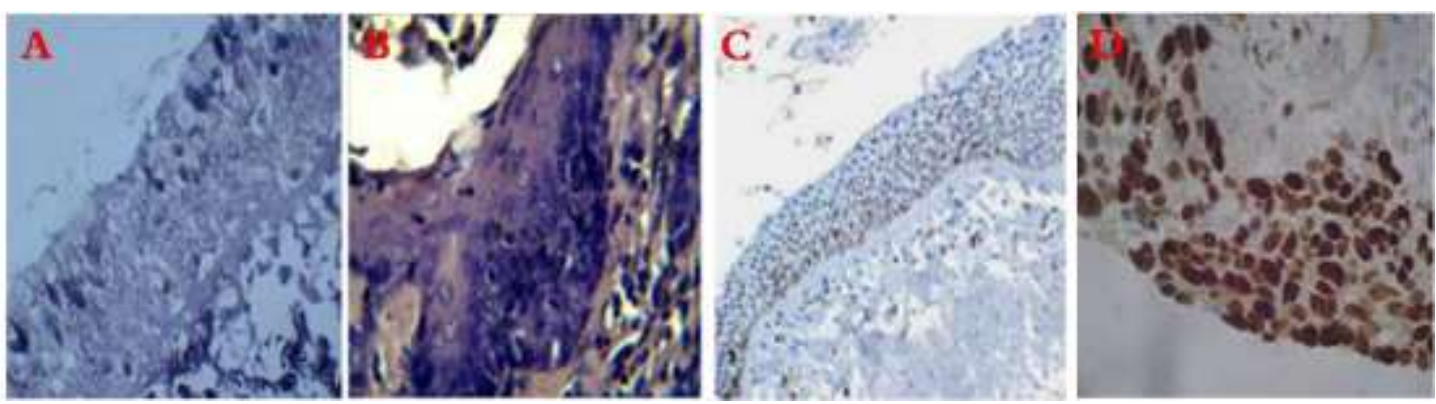

Fig. 3. In situ hybridization assays show MDM2 mRNA and protein expressions in rat lung tissues. A and B. MDM2 mRNA expression $(\times 400)$; C and D. MDM2 protein expression $(\times 400)$. 